\title{
JSH Statement: Asahikawa declaration in promotion of diversity by the Japanese society of hypertension-the JSH Asahikawa declaration
}

\author{
Satoko Nakamura ${ }^{1}$ Naoyuki Hasebe ${ }^{2} \cdot$ Midori Yatabe $^{3} \cdot$ Takuya Kishi $^{4} \cdot$ Mari Ishida $^{5}$ - Atsuhiro Ichihara ${ }^{3}$. \\ Koichi Node $^{6} \cdot$ Toshihiko Ishimitsu $^{7} \cdot$ Hiroshi Itoh $^{8} \cdot$ Masaaki Ito $^{9} \cdot$ Mitsuru Ohishi $^{10} \cdot$ Takayoshi Ohkubo $^{11}$. \\ Hisashi Kai ${ }^{12}$ - Naoki Kashihara ${ }^{13} \cdot$ Kazuomi Kario $^{14} \cdot$ Shigeyuki Saitoh $^{15}$ - Takuya Tsuchihashi $^{16}$. Akira Nishiyama ${ }^{17}$. \\ Jitsuo Higaki ${ }^{18} \cdot$ Katsuyuki Miura $^{19} \cdot$ Hiromi Rakugi $^{20} \cdot$ Yuhei Kawano $^{21} \cdot$ Kouichi Tamura $^{22} \cdot$ Takashi Yatabe $^{23}$. \\ Yusuke Ohya ${ }^{24} \cdot$ Sadayoshi Ito ${ }^{25}$
}

Received: 15 March 2019 / Accepted: 24 March 2019 / Published online: 8 April 2019

(c) The Japanese Society of Hypertension 2019

The Japanese Society of Hypertension (JSH) has a longstanding history of cooperation among doctors and allied health professionals across diverse generations, gender, and specialties. In addition, the JSH has promoted career development and lifelong education of its members, appointing young and/or women investigators to session chairs and symposium speakers. The JSH now strives for globalization of the organization and its meetings, working in close relationship with Asian and Western friends and counterparts [1].

To further advance these achievements, the JSH aims to be a society where people of different backgrounds can equally come together to contribute. The goal of this declaration is to create a system and environment for hypertension practice in which all its members can achieve their full potential, overcoming differences in generation, gender, nationality, religious believes, physical and mental characteristics, and sexual preferences, while being assured that their personal dignity and values are respected. This may be realized as public awareness programs organized cooperatively by citizens and multidisciplinary medical personnel or improved physician-patient relationship with mutually

Asahikawa Declaration in Promotion of Diversity by the Japanese Society of Hypertension-the JSH Asahikawa Declaration-was announced at the 41st Annual Scientific Meeting of the Japanese Society of Hypertension, which was held in Asahikawa on 14-16 September 2018.

Satoko Nakamura

s-nakamura@tamateyama.ac.jp

Extended author information available on the last page of the article understandable hypertension management practice based on the philosophy of diversity and empathy. Such initiatives are what the JSH considers as its true social responsibility and contribution.

The JSH has announced "Asahikawa Declaration in Promotion of Diversity by the Japanese Society of Hypertension-the JSH Asahikawa Declaration," at its 41st Annual Scientific Meeting in Asahikawa, Hokkaido. In association with the JSH Future Plan [2], we pledge to undertake a specific action plan comprised of the following 5 key missions to achieve our goal of this declaration.

The JSH will:

1) Advocate the spirit of diversity to value heterogeneity and acknowledge differences in all patients and medical professionals.

2) Support the fostering of proper educational, research, and clinical practice environment to realize work-life integration while maximizing the competencies with diversity.

3) Respect individuality of patients, promoting awareness programs and practice style to make hypertension management plainer and seamless.

4) Create a supporting system for society members who need reasonable accommodation with respect.

5) Promote a diverse and inclusive work environment where diversity is valued.

Acknowledgements The authors thank the members of the Diversity and Inclusion Committee of the Japanese Society of Hypertension, including Kimika Arakawa, Yuki Imaizumi, Kei Kamide, Mai Kabayama, Miho Kusaka, Setsuko Kuroda, Mariyo Sakoda, Akiyo Tanabe, Kensuke Noma, Ako Fukami, Megumi Fujita, Asako Mito, Junichi Yatabe, Sumiyo Yamashita, Sumiko Yoshida, Sanae Watanabe, for their help in developing this declaration. 


\section{Compliance with ethical standards}

Conflict of interest The authors declare that they have no conflict of interest.

Publisher's note: Springer Nature remains neutral with regard to jurisdictional claims in published maps and institutional affiliations.

\section{References}

1. Ito M, Ishimitsu T, Ichihara A, Itoh $\mathrm{H}$, Ohishi M, Ohkubo T, et al. JSH Statement: Kyoto declaration on hypertension research in Asia. Hypertens Res. 2019. https://doi.org/10.1038/s41440-018-0172-8.

2. Node K, Kishi T, Tanaka A, Itoh H, Rakugi H, Ohya Y, et al. The Japanese Society of Hypertension-Digest of plan for the future. Hypertens Res. 2018;41:989-90.

\section{Affiliations}

Satoko Nakamura ${ }^{1} \cdot$ Naoyuki Hasebe ${ }^{2} \cdot$ Midori Yatabe $^{3} \cdot$ Takuya Kishi $^{4} \cdot$ Mari Ishida $^{5} \cdot$ Atsuhiro Ichihara $^{3}$. Koichi Node ${ }^{6} \cdot$ Toshihiko Ishimitsu $^{7} \cdot$ Hiroshi Itoh $^{8} \cdot$ Masaaki Ito $^{9} \cdot$ Mitsuru Ohishi $^{10} \cdot$ Takayoshi Ohkubo $^{11}$. Hisashi Kai ${ }^{12} \cdot$ Naoki Kashihara $^{13} \cdot$ Kazuomi Kario $^{14} \cdot$ Shigeyuki Saitoh $^{15} \cdot$ Takuya Tsuchihashi $^{16} \cdot$ Akira Nishiyama $^{17}$. Jitsuo Higaki ${ }^{18}$ - Katsuyuki Miura ${ }^{19}$ - Hiromi Rakugi ${ }^{20}$ - Yuhei Kawano ${ }^{21}$ - Kouichi Tamura ${ }^{22}$ - Takashi Yatabe ${ }^{23}$. Yusuke Ohya ${ }^{24}$. Sadayoshi Ito ${ }^{25}$

1 Department of Nutritional Sciences for Well-being, Kansai University of Welfare Sciences, Kashiwara, Japan

2 Division of Cardiology, Nephrology, Pulmonology and Neurology, Department of Internal Medicine, Asahikawa Medical University, Asahikawa, Japan

3 Department of Endocrinology and Hypertension, Tokyo Women's Medical University, Tokyo, Japan

4 Department of Advanced Risk Stratification for Cardiovascular Diseases, Center for Disruptive Cardiovascular Medicine, Kyushu University, Fukuoka, Japan

5 Department of Cardiovascular Physiology and Medicine, Graduate School of Biomedical and Health Sciences, Hiroshima University, Hiroshima, Japan

6 Department of Cardiovascular Medicine, Saga University, Saga, Japan

7 Department of Cardiology and Nephrology, Dokkyo Medical University Hospital, Mibu, Japan

8 Department of Endocrinology, Metabolism and Nephrology, School of Medicine, Keio University, Tokyo, Japan

9 Department of Cardiology and Nephrology, Mie University Graduate School of Medicine, Tsu, Japan

10 Department of Cardiovascular Medicine and Hypertension, Graduate School of Medical and Dental Sciences, Kagoshima University, Kagoshima, Japan

11 Department of Hygiene and Public Health, Teikyo University School of Medicine, Tokyo, Japan

12 Department of Cardiology, Kurume University Medical Center, Kurume, Japan
13 Department of Nephrology and Hypertension, Kawasaki Medical School, Kurashiki, Japan

14 Division of Cardiovascular Medicine, Department of Medicine, Jichi Medical University School of Medicine, Shimotsuke, Japan

15 School of Health Sciences, Sapporo Medical University, Sapporo, Japan

16 Hypertension Center, Steel Memorial Yawata Hospital, Kitakyushu, Japan

17 Department of Pharmacology, Kagawa University Medical School, Kagawa, Japan

18 Minami-Matsuyama Hospital, Matsuyama, Japan

19 Department of Public Health, Shiga University of Medical Science, Otsu, Japan

20 Department of Geriatric and General Medicine, Osaka University Graduate School of Medicine, Suita, Japan

21 Department of Medical Technology, Teikyo University Fukuoka, Omuta, Japan

22 Department of Medical Science and Cardio-Renal Medicine, Yokohama City University Graduate School of Medicine, Yokohama, Japan

23 Yatabe and Momii Law Office, Tokyo, Japan

24 Department of Cardiovascular Medicine, Nephrology and Neurology, University of the Ryukyus Graduate School of Medicine, Okinawa, Japan

25 Division of Nephrology, Endocrinology and Hypertension, Tohoku University Graduate School of Medicine, Sendai, Japan 